\title{
Comparison of two threshold detection criteria methodologies for determination of probe positivity for intraoperative in situ identification of presumed abnormal ${ }^{18} \mathrm{~F}$-FDG-avid tissue sites during radioguided oncologic surgery
}

Gregg J Chapman ${ }^{1+}$, Stephen P Povoski ${ }^{2^{*}+}$, Nathan C Hall ${ }^{3}$, Douglas A Murrey $\mathrm{Jr}^{3}$, Robert Lee and Edward W Martin $\mathrm{Jr}^{2}$

\begin{abstract}
Background: Intraoperative in situ identification of ${ }^{18} \mathrm{~F}$-FDG-avid tissue sites during radioguided oncologic surgery remains a significant challenge for surgeons. The purpose of our study was to evaluate the 1.5-to-1 ratiometric threshold criteria method versus the three-sigma statistical threshold criteria method for determination of gamma detection probe positivity for intraoperative in situ identification of presumed abnormal ${ }^{18} \mathrm{~F}$-FDG-avid tissue sites in a manner that was independent of the specific type of gamma detection probe used.

Methods: From among 52 patients undergoing appropriate in situ evaluation of presumed abnormal ${ }^{18} \mathrm{~F}$-FDG-avid tissue sites during ${ }^{18} \mathrm{~F}$-FDG-directed surgery using 6 available gamma detection probe systems, a total of 401 intraoperative gamma detection probe measurement sets of in situ counts per second measurements were cumulatively taken.

Results: For the 401 intraoperative gamma detection probe measurement sets, probe positivity was successfully met by the 1.5-to-1 ratiometric threshold criteria method in 150/401 instances (37.4\%) and by the three-sigma statistical threshold criteria method in 259/401 instances (64.6\%) $(P<0.001)$. Likewise, the three-sigma statistical threshold criteria method detected true positive results at target-to-background ratios much lower than the 1.5-to-1 target-to-background ratio of the 1.5-to-1 ratiometric threshold criteria method.

Conclusions: The three-sigma statistical threshold criteria method was significantly better than the 1.5-to-1 ratiometric threshold criteria method for determination of gamma detection probe positivity for intraoperative in situ detection of presumed abnormal ${ }^{18} \mathrm{~F}$-FDG-avid tissue sites during radioguided oncologic surgery. This finding may be extremely important for reshaping the ongoing and future research and development of gamma detection probe systems that are necessary for optimizing the in situ detection of radioisotopes of higher-energy gamma photon emissions used during radioguided oncologic surgery.
\end{abstract}

Keywords: ${ }^{18} \mathrm{~F}$-fluorodeoxyglucose, ${ }^{18} \mathrm{~F}-\mathrm{FDG}$, Target-to-background ratio, Tumor-to-background ratio, Three-sigma, Ratiometric, Threshold, ${ }^{18} \mathrm{~F}$-FDG-directed surgery, Radioguided surgery, Intraoperative detection, Gamma detection probes, in situ, Count rate, Neoplasms, Oncologic

\footnotetext{
* Correspondence: stephen.povoski@osumc.edu

${ }^{\dagger}$ Equal contributors

${ }^{2}$ Division of Surgical Oncology, Department of Surgery, Arthur G. James

Cancer Hospital and Richard J. Solove Research Institute and Comprehensive

Cancer Center, The Ohio State University Wexner Medical Center, Columbus,

$\mathrm{OH}$ 43210, USA

Full list of author information is available at the end of the article
}

C Biomed Central
(C) 2014 Chapman et al.; licensee BioMed Central Ltd. This is an Open Access article distributed under the terms of the Creative Commons Attribution License (http://creativecommons.org/licenses/by/4.0), which permits unrestricted use, distribution, and reproduction in any medium, provided the original work is properly credited. The Creative Commons Public Domain Dedication waiver (http://creativecommons.org/publicdomain/zero/1.0/) applies to the data made available in this article, unless otherwise stated. 


\section{Background}

The use of intraoperative gamma probe detection technology has become a mainstay in the surgical management of many solid malignancies [1]. While current commerciallyavailable gamma detection probe technology works well for detecting radioisotopes of low-energy gamma photon emissions, such as ${ }^{99 \mathrm{~m}} \mathrm{Tc}(140$ and $142 \mathrm{KeV})$, the detection of radioisotopes of higher-energy gamma photon emissions, such as ${ }^{18} \mathrm{~F}$-fluorodeoxyglucose $\left({ }^{18} \mathrm{~F}\right.$-FDG), which is based upon $511 \mathrm{KeV}$ gamma emissions following positron annihilation, remains a significant challenge for surgeons [1,2]. With increasing experience in ${ }^{18} \mathrm{~F}$-FDGdirected surgery techniques [1-45], multiple investigators have recognized the challenges related to the intraoperative in situ identification presumed abnormal ${ }^{18}$ F-FDG-avid tissue sites during radioguided oncologic surgery [2,8,14,18,19,21,28,46-49].

The most significant challenge related to successful intraoperative in situ identification of presumed abnormal ${ }^{18}$ F-FDG-avid tissue sites during ${ }^{18}$ F-FDG-directed surgery is the particular situation of encountering a resultant low target-to-background ratio from the radiation emissions of ${ }^{18}$ F-FDG $[2,8,14,18,19,21,28,46-49]$. It has been suggested by some authors that a minimum in situ targetto-background ratio of 1.5 -to-1 for ${ }^{18}$ F-FDG is necessary for allowing the surgeon to comfortably differentiate tumor-bearing tissues from that of normal tissue during ${ }^{18}$ F-FDG-directed surgery $[14,18,19]$. However, this target-to-background ratio represents an arbitrary and fixed ratio determination that can be affected by multiple factors, including ${ }^{18}$ F-FDG uptake by tumor-bearing tissues, the distribution and degree of background radiation within various surrounding tissues which do not represent tumor-bearing tissues, and innumerable factors related to the technical specifications of the specific detection probe system used for making counts per second measurements. Our own experience with ${ }^{18}$ F-FDGdirected surgery $[1-5,20,22-27,29,31,41]$ has shown us that the observed in situ target-to-background ratio of presumed abnormal ${ }^{18} \mathrm{~F}$-FDG-avid tissue is commonly less than 1.5-to-1, and as previously stated is highly dependent upon the specific detection probe system [2]. As a result, when intraoperative detection of in situ ${ }^{18}$ F-FDG-avid tissue sites relies solely on a fixed target-to-background ratio (i.e., ratiometric threshold method) as the threshold for probe positivity, the success of such detection methods can be limited and resultantly frustrating for the surgeon [2]. Therefore, we have suggested that improved intraoperative in situ identification of ${ }^{18}$ F-FDG-avid tissue sites can be accomplished by the use of the three-sigma statistical threshold criteria method for determination of gamma detection probe positivity [2].

The three-sigma statistical threshold criteria for determination of gamma detection probe positivity has been previously well characterized by Thurston [2,50-53], and has been previously well-utilized for radioimmunoguided surgery involving ${ }^{125}$ I-labeled anti-TAG-72 monoclonal antibodies [2,50-58]. The three-sigma statistical threshold criteria defines any given tissue as being probe positive when the count rate in that tissue exceeds three standard deviations above the mean count rate detected within normal adjacent tissue [2,50-58].

To further investigate our contention that improved intraoperative in situ identification of ${ }^{18}$ F-FDG-avid tissue sites can be accomplished by the use of the three-sigma statistical threshold criteria method for determination of gamma detection probe positivity, we recently evaluated the success rate of intraoperative in situ detection of presumed abnormal ${ }^{18}$ F-FDG-avid tissue sites using the 1.5-to-1 ratiometric threshold criteria method and the three-sigma statistical threshold criteria method for three different gamma detection probe systems in a limited data set [2]. This limited data set consisted of a group of seven patients, representing a total of nine separate ${ }^{18} \mathrm{~F}$-FDG-avid tissue sites, in which all ${ }^{18}$ F-FDG-avid tissue sites were identified by same-day preoperative diagnostic positron emission tomography/computed tomography (PET/CT) imaging, were intraoperatively assessed in situ with all three gamma detection probe systems, and were subsequently surgically excised. In this analysis, we found that successful intraoperative in situ detection of ${ }^{18}$ F-FDG-avid tissue sites was more frequently accomplished by using the three-sigma statistical threshold criteria method than by using the 1.5-to-1 ratiometric threshold criteria method with each of the three gamma detection probe systems tested. Nevertheless, due to the small sample size of our limited data set that was available for the $2 \times 3$ contingency table analysis, there was no significant difference in our statistical analysis of overall comparison of the three gamma detection probe systems utilized as a function of the specific threshold criteria method for the determination of gamma detection probe positivity [2].

In the current analysis, and in order to rectify the above problem faced in our previous report regarding limited sample size availability for statistical analysis [2], we purposefully chose to examine the effects of utilizing the 1.5-to-1 ratiometric threshold criteria method versus the three-sigma statistical threshold criteria method for determination of gamma detection probe positivity for intraoperative in situ identification of presumed abnormal ${ }^{18}$ F-FDG-avid tissue sites within our entire study population in a manner that was completely independent of the specific type of gamma detection probe system used. As a result, this particular approach allowed for a much larger number of intraoperative gamma detection probe measurement sets that were available for the statistical analysis. 


\section{Methods}

Logistics for deriving the complete data set from individual intraoperative gamma detection probe measurement sets of in situ counts

The data analyzed herein were obtained from the master database of a Cancer IRB-approved, prospective, pilot study protocol (approved by The Ohio State University Cancer IRB) involving patients undergoing ${ }^{18}$ F-FDG-directed surgery for known or suspected malignancy at the Arthur G. James Cancer Hospital and Richard J. Solove Research Institute of The Ohio State University Wexner Medical Center. Sixty-five patients originally gave informed consent to participate in the Cancer IRB-approved, prospective, pilot study protocol, of which 60 patients were taken to the operating room, of which 58 patients underwent ${ }^{18}$ F-FDG-directed surgery using available gamma detection probes, and of which 52 patients underwent appropriate in situ evaluation of presumed abnormal ${ }^{18} \mathrm{~F}$-FDG-avid tissue sites (i.e., measurements taken before any such tissue was surgically excised) using various combinations of 6 different available gamma detection probe systems. Thus, 6 of the 58 patients who underwent ${ }^{18}$ F-FDG-directed surgery using available gamma detection probes did not have in situ evaluation of presumed abnormal ${ }^{18}$ F-FDG-avid tissue sites, and those 6 patients only underwent ex situ evaluation of presumed abnormal ${ }^{18}$ F-FDG-avid tissue sites (i.e., measurements taken after any such tissue was surgically excised). Additionally, none of the in situ gamma detection probe measurement sets in the 52 patients undergoing appropriate in situ evaluation of presumed abnormal ${ }^{18}$ F-FDG-avid tissue sites using various combinations of 6 different available gamma detection probe systems were excluded from the analysis.

Ninety-seven separate sites were selected based on identification of finite areas of presumed abnormal ${ }^{18} \mathrm{~F}$-FDGavidity seen on a preoperative diagnostic ${ }^{18}$ F-FDG PET/CT scan from the 52 patients described who eventually underwent appropriate in situ evaluation of presumed abnormal ${ }^{18}$ F-FDG-avid tissue sites. All presumed abnormal ${ }^{18}$ F-FDG-avid tissue sites were defined as abnormal as based upon the reading and official radiology report issued on the preoperative diagnostic ${ }^{18}$ F-FDG PET/CT scan by the reporting attending nuclear medicine physician and were not defined as abnormal as based upon any specific predetermined cut-off level for the standardized uptake value (SUV) seen in those ${ }^{18} \mathrm{~F}$-FDG-avid tissue sites. Intraoperatively, these 97 in situ presumed abnormal ${ }^{18}$ F-FDGavid sites were evaluated with various combinations of 6 available gamma detection probe systems in these 52 patients. As a result, 401 intraoperative gamma detection probe measurement sets of in situ counts per second measurements were cumulatively taken from among the 6 available gamma detection probe systems, representing the complete data set used in our current statistical analyses.
These 6 available gamma detection probe systems included 4 commercially-available gamma detection probe systems and 2 prototype experimental probe designs. The 4 commercially-available gamma detection probe systems consisted of 2 commercially-available gamma detection probe systems which were originally designed to detect lower energy gamma-emitting radioisotopes (Neoprobe ${ }^{\odot}$ 1000 and Neoprobe ${ }^{\oplus}$ 2000, formally Neoprobe Corporation, Dublin; Ohio) and 2 commercially-available gamma detection probe systems which were specifically designed in an attempt to detect $511 \mathrm{KeV}$ high-energy gamma emissions from ${ }^{18}$ F-FDG positron annihilations (Neoprobe ${ }^{\odot}$ High Energy Probe, formally Neoprobe Corporation, Dublin; Ohio; and RMD Navigator GPS ${ }^{\mathrm{TM}}$ Gamma-PET $^{\mathrm{TM}}$ Probe, formally RMD Instruments, Watertown, Massachusetts). The 2 prototype experimental probe designs consisted of: (1) a modified version of a lower energy gamma-emitting radioisotopes-detecting commercially-available probe, retro-fitted with a tungsten collimator of sufficient thickness to block $90 \%$ of $511 \mathrm{KeV}$ gamma radiation, and which was intended to limit the field-of-view of the detection probe in order to increase the target-to-background ratio; and (2) an experimental detection probe which incorporated an $\mathrm{x}$-ray fluorescence element to transfer the $511 \mathrm{KeV}$ high-energy gamma radiation to the K-alpha x-ray energy of the element (i.e., $72 \mathrm{KeV}$ ), which allowed for this lower K-alpha $\mathrm{x}$-ray energy to be counted at a higher efficiency than the 511 $\mathrm{KeV}$ high-energy gamma emissions [2,59].

The entire group of 401 intraoperative gamma detection probe measurement sets of in situ counts per second measurements collected from our entire study population were cumulatively analyzed in a manner that was completely independent of the specific type of gamma detection probe system used to evaluate gamma detection probe positivity for intraoperative in situ identification of presumed abnormal ${ }^{18}$ F-FDG-avid tissue sites by the 1.5 -to- 1 ratiometric threshold criteria method and by the three-sigma statistical threshold criteria method.

\section{Definition of each intraoperative gamma detection probe measurement sets of in situ counts}

As previously reported [2], for each intraoperative gamma detection probe measurement set, an averaged count rate (i.e., counts per second) was taken from an area selected for the in situ measurement of background tissue count rate, and from the area of presumed abnormal ${ }^{18}$ F-FDG-avid tissue selected for the in situ measurement of target tissue count rate. An area of presumed normal tissue within a region adjacent to the area of the target tissue was selected for the measurement of background tissue count rate. Three separate recorded values were used to generate each averaged target tissue count rate measurement determined for each area of presumed abnormal ${ }^{18} \mathrm{~F}$-FDG-avid tissue. All values used for the averaged count rate measurements 
were normalized and reported as averaged counts per second. Count rate normalization consists of dividing acquired counts by the count duration in seconds. It is important to emphasize that all of the averaged target tissue count rate measurements that were reported in this paper represented measurements taken on an area of presumed abnormal ${ }^{18} \mathrm{~F}$-FDG-avid tissue before any such tissue was surgically excised (i.e., in situ measurements). None of the averaged target tissue count rate measurements that were reported in this paper represented measurements taken on an area of presumed abnormal ${ }^{18}$ F-FDG-avid tissue after any such tissue was surgically excised (i.e., ex situ measurements).

\section{Rationale for recording in situ target tissue count rate measurements for the intraoperative identification of presumed abnormal ${ }^{18} \mathrm{~F}$-FDG-avid tissue sites}

A major inherent limitation in performing PET/CT oncologic imaging with ${ }^{18} \mathrm{~F}$-FDG is related to the fact that ${ }^{18} \mathrm{~F}$-FDG is not a cancer-specific imaging agent, and for which ${ }^{18}$ F-FDG readily accumulates within tissues representing benign disease processes (i.e., infection, inflammation, and trauma) and ${ }^{18}$ F-FDG readily accumulates within various normal tissues (i.e., brain, heart, mucosa and smooth muscle of the stomach, small intestines and colon, thyroid, liver, spleen, kidneys, ureters, and bladder) that have a typical physiologic propensity for ${ }^{18}$ F-FDG accumulation [1,2]. These sites of benign disease accumulation and physiologic accumulation of ${ }^{18} \mathrm{~F}$-FDG can result in an intrinsically high level of background ${ }^{18}$ F-FDG activity within regions of presumed normal tissues that may co-exist with presumed abnormal ${ }^{18}$ F-FDG-avid tissue sites. This can be particularly challenging when any given presumed abnormal ${ }^{18} \mathrm{~F}$-FDGavid tissue site has a relatively low target site count rate. Resultantly, recording the in situ measurements for the averaged target tissue count rate on any given area of presumed abnormal ${ }^{18} \mathrm{~F}$-FDG-avid tissue before any such tissue was surgically excised would represent the worst-case scenario as related to the intrinsic background activity of ${ }^{18}$ F-FDG. Conversely, recording the ex situ measurements for the averaged target tissue count rate on any given area of presumed abnormal ${ }^{18} \mathrm{~F}$-FDG-avid tissue after any such tissue was surgically excised would represent the best-case scenario as related to the intrinsic background activity of ${ }^{18}$ F-FDG. Therefore, to most definitively determine whether there was any significant difference between these two threshold detection criteria methodologies (i.e., the 1.5-to-1 ratiometric threshold criteria method and the three-sigma statistical threshold criteria method) for determination of probe positivity during attempted identification of presumed abnormal ${ }^{18} \mathrm{~F}-\mathrm{FDG}$-avid tissue sites during radioguided oncologic surgery, we selected the worst-case scenario as related to the intrinsic background ${ }^{18} \mathrm{~F}$-FDG activity and elected to record the in situ measurements for the averaged target tissue count rate on all presumed abnormal ${ }^{18}$ F-FDG-avid tissue site before any such tissue sites were surgically excised, in order to maximize the negative impact of the intrinsic background ${ }^{18}$ F-FDG activity.

\section{Definition and determination of the 1.5-to-1 target-to-background ratio method for probe positivity}

A target-to-background ratio was calculated for each target tissue as defined as the ratio of the averaged target tissue count rate to the background tissue count rate. The ratiometric threshold of 1.5 -to-1 or greater was set as the ratiometric threshold criteria for probe positivity (i.e., 1.5-to-1 ratiometric threshold criteria method).

\section{Definition and theoretical derivation of the three-sigma} statistical threshold criteria method of probe positivity For each intraoperative target tissue measurement, a three-sigma statistical threshold criteria count rate was calculated by the methodology popularized of Thurston [2,50-53]. The three-sigma statistical threshold criteria was determined from an area of presumed normal background tissue by multiplying the standard deviation of the normal background count rate in an area of presumed normal background tissue by a factor of three, and then adding that calculated number to the mean value of the normal background count rate. The condition for probe positivity was met if the count rate for the target tissue (i.e., target count rate) exceeded the calculated three-sigma criteria count rate threshold.

The three-sigma statistical threshold criteria represents a specific application of binary hypothesis testing. The theoretical deviation of the three-sigma statistical threshold criteria as it relates to radioactivity has its origins in the classical work of Currie [60]. In the classical application to radioactivity by Currie [60], the presence of a radiation source is tested against the null hypothesis that no source is present in excess of the background radiation. The resulting threshold for the alternative hypothesis, i.e. that there is a source present, can be reduced to a single variable, $\mu_{B}$, the mean value of the background radiation count. This threshold for accepting the alternative hypothesis $H_{1}$ takes the form:

$$
\boldsymbol{H}_{1} \geq \boldsymbol{\mu}_{\boldsymbol{B}}+\boldsymbol{K}_{1}+\boldsymbol{K}_{2} * \sqrt{\boldsymbol{\mu}_{\boldsymbol{B}}}
$$

where $K_{1}$ and $K_{2}$ are constants. For counts rates exceeding 30 per second, $K_{1}$ is considered negligibly small and the threshold for accepting the alternative hypothesis is

$$
\boldsymbol{\mu}_{\boldsymbol{B}}+\boldsymbol{K}_{2} * \sqrt{\boldsymbol{\mu}_{\boldsymbol{B}}}
$$

In the current application, the presumed ${ }^{18} \mathrm{~F}$-FDG-avid tissue site contains activity from both background radiation 
and the source radiation. To derive the preceding relationship, the combined count rates must be taken into account. Although the following derivation is applied to raw counts, the same analysis can be applied to count rate measurements with no loss of generality if the count rates are calculated using equal time intervals of raw count measurements, and provided that the raw counts are sufficient to support the statistical analysis without introducing error secondary to insufficient sample size.

Radioactive decay is a Poisson distributed discrete random process since each event is independent of the preceding event. If the number of counts in a Poisson distribution exceeds approximately 30 , a continuous Gaussian distribution provides a close approximation [61]. However, the value for standard deviation used in the Gaussian approximation must be equal to the square root of the mean value since this is a characteristic of the original Poisson distribution. If this condition is imposed, the two distributions can be used interchangeably and Z-score statistics can be applied using the one-sided Gaussian approximation. Counts are discrete values in both the Poisson and the corresponding Gaussian approximation.

Consider the two probability distributions of counts illustrated in Figure 1, with one count distribution representing the background count measurement, and with the second count distribution representing the target count measurement. By definition, the target count measurement $(\boldsymbol{T})$ is the summation of source count measurement $(S)$ plus the background count measurement $(\boldsymbol{B})$. Since the source count is the difference between the target count and background count, the variance of the source count is the sum of the variances of the target count and the background count.

$$
\sigma_{S}^{2}=\sigma_{T}^{2}+\sigma_{B}^{2}
$$

When no source activity is present, the variance for the target is equal to the variance for the background and:

$$
\sigma_{S}=\sqrt{2 \sigma_{B}^{2}}
$$

The Critical Limit $\left(\mathrm{L}_{\mathrm{C}}\right)$ for false positive counts is based on the one-sided $\mathrm{Z}$ parameter. A value of $\mathrm{K}$ is chosen for the desired percentile of statistical certainty above the mean background count. $\mathrm{K}$ times the standard deviation of the source count distribution defines the $L_{C}$ at the desired percentile such that:

$$
\begin{aligned}
& L_{C}=\mu_{B}+K \sigma_{S} \\
& L_{C}=\mu_{B}+K \sqrt{2} \sigma_{B}
\end{aligned}
$$

If the same $\mathrm{K}$ parameter is chosen to define an equal percentile of false negative and false positive probabilities, the $\mathrm{L}_{\mathrm{C}}$ will also coincide with:

$$
\begin{aligned}
& L_{C}=\mu_{D^{-}} K \sigma_{D} \\
& \mu_{D}=L_{C}+K \sigma_{D}
\end{aligned}
$$

where $\mu_{D}$ is the desired source count corresponding to the minimum detectable count difference.

The standard deviation for the minimum detectible distribution, $\sigma_{D}$, can also be expressed as a function of the standard deviation for both the background count

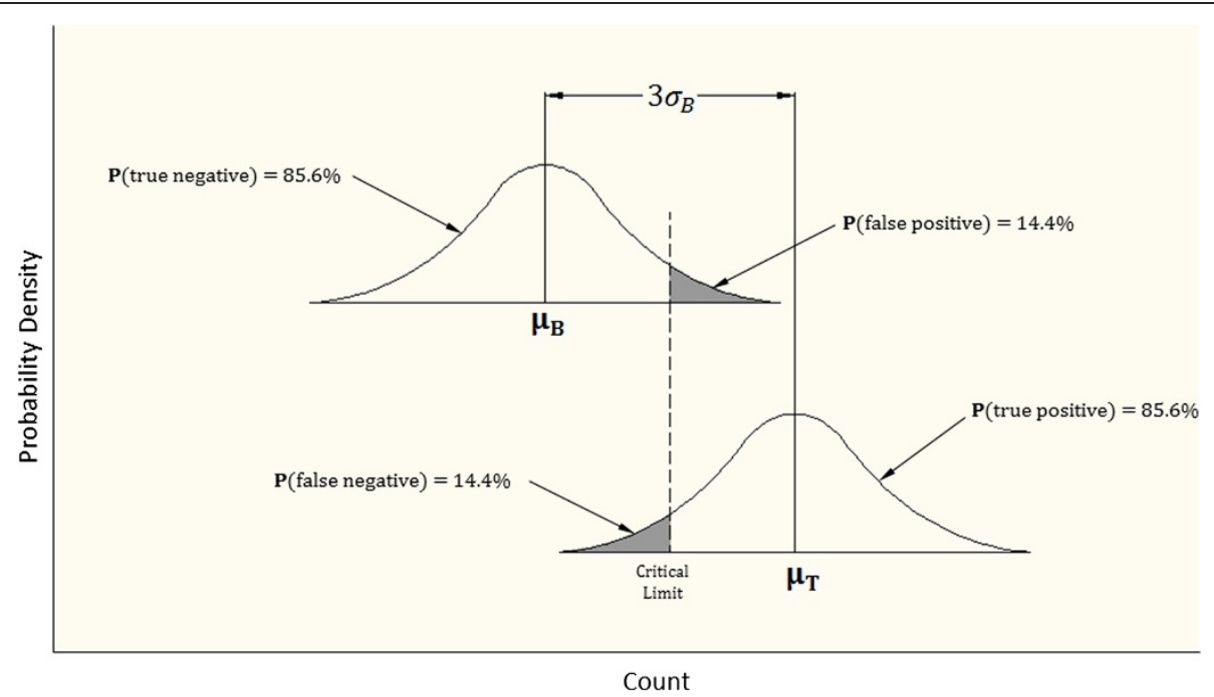

Figure 1 Illustration of the probability distribution for the background count measurement and the target count measurement. The target count measurement $(\boldsymbol{T})$ represents the summation of source count measurement $(\boldsymbol{S})$ plus the background count measurement $(\boldsymbol{B})$. Probabilities $(\boldsymbol{P})$ for true positive, true negative, false positive, and false negative are shown for target count rates at three standard deviations above the mean background count. 
and target count. Substituting the square root mean count values for the standard deviations reduces the following expression.

$$
\begin{aligned}
\sigma_{D} & =\sqrt{\sigma_{T}^{2}+\sigma_{B}^{2}}=\sqrt{\mu_{T}+\mu_{B}} \\
& =\sqrt{\mu_{D}+\mu_{B}+\mu_{B}}=\sqrt{\mu_{D}+2 \mu_{B}}
\end{aligned}
$$

Since $\mu_{D}=L_{C}+K \sigma_{D}$, the $\mathrm{L}_{C}$ as a function of the mean background count can be substituted.

$$
\mu_{D}=\left(\mu_{B}+K \sqrt{2} \sigma_{B}\right)+K \sqrt{\mu_{D}+2 \mu_{B}}
$$

This equation can be algebraically reduced to:

$$
\boldsymbol{\mu}_{\boldsymbol{D}}=\boldsymbol{\mu}_{\boldsymbol{B}}+\boldsymbol{K}^{2}+\boldsymbol{K} 2 \sqrt{2} * \sqrt{\boldsymbol{\mu}_{\boldsymbol{B}}}
$$

$K^{2}$ (a Z-table value) is less than 3 for all certainty levels less than $95.6 \%$, and can be considered negligible for mean background counts greater than 30. This reduces the minimum detectable count threshold to:

$$
\boldsymbol{\mu}_{D}=\boldsymbol{\mu}_{B}+(\boldsymbol{K} 2 \sqrt{2}) * \boldsymbol{\sigma}_{B}
$$

Calculating the percentile for the three-sigma statistical threshold criteria:

$$
\begin{aligned}
& 3=K 2 \sqrt{2} \\
& \therefore K=\frac{3}{2 \sqrt{2}}=1.0607
\end{aligned}
$$

The one-sided percentile calculation for a Z-score of 1.0607 is approximately equal to $85.6 \%$ [62].

Thus, for the three-sigma statistical threshold criteria, the probability for either a false positive or false negative finding is approximately $14.4 \%$. The confidence level for probe positivity is $85.6 \%$. Comparing the distribution of the background count and the target count in Figure 1 illustrates the relationship between these probabilities. The probabilities of false positive and false negative findings are calculated as the area under the curve in the shaded regions indicated in Figure 1.

\section{Statistical analysis}

The software program IBM SPSS 21 for Windows ${ }^{\bullet}$ (SPSS, Inc., Chicago, Illinois) was used for all data analyses. All continuous variable results were expressed as mean $( \pm$ SD, range). The probe positivity comparison for the 1.5-to-1 ratiometric threshold criteria method versus the three-sigma statistical threshold criteria method was made by way of a $2 \times 2$ contingency table analysis using the Pearson chi-square test. The P-value for the Pearson chi-square test comparison was reported as an exact two-sided P-value. A P-value of 0.05 or less was considered to be statistically significant.
The drawing software program AutoCAD LT 2010 (AutoDesk, Inc., San Rafael, California) was used for the generation of Figure 1.

The software program MATLAB R2013b version 8.2.0.701 (MathWorks, Natick, Massachusetts) was used for the generation of Figures 2, 3, and 4 .

\section{Results and discussion}

Of the 52 patients undergoing in situ evaluation of presumed abnormal ${ }^{18} \mathrm{~F}$-FDG-avid tissue sites using any given gamma detection probe system at the time of ${ }^{18} \mathrm{~F}$-FDG-directed surgery, there were 30 Caucasian females, 18 Caucasian males, 2 African-American female, 1 African-American male, and 1 Asian female. The mean patient age was $58( \pm 10$, range $37-83)$ years. The mean patient body weight was $81.7( \pm 20.2$, range $43.5-142.9)$ kilograms. The mean patient same-day pre-scanning blood sugar level of $106( \pm 18$, range 78-157) milligrams per deciliter. The mean ${ }^{18}$ F-FDG injection dose was 537 ( \pm 55 , range 387-611) megabecquerels. The mean time from ${ }^{18}$ F-FDG injection to the time of the start of surgery was $242( \pm 82$, range 108-449) minutes. The mean time from ${ }^{18} \mathrm{~F}$-FDG injection to the time of the start of appropriate in situ evaluation of presumed abnormal ${ }^{18} \mathrm{~F}$-FDG-avid tissue sites using any of the given gamma detection probe system was 278 ( \pm 88 , range $145-501)$ minutes.

The mean of the averaged target tissue count rate for the 401 gamma detection probe measurement sets was $2,047( \pm 3,706$, range $6-16,353)$ counts per second. The mean of the background tissue count rate in an area of presumed normal tissue within a region adjacent to the presumed ${ }^{18} \mathrm{~F}$-FDG-avid tissue site for the 401 gamma detection probe measurement sets was $1,709( \pm 3,172$, range 4-13,020) counts per second. The mean of the calculated 1.5-to-1 target-to-background ratio for the 401 gamma detection probe measurement sets was 1.53 $( \pm 0.93$, range $0.12-9.88$ ). The mean of the calculated three-sigma statistical threshold criteria count rate for the 401 gamma detection probe measurement sets was $1,796$ ( $\pm 3,257$, range $11-13,362)$ counts per second.

For the 401 gamma detection probe measurement sets, probe positivity was successfully met by the 1.5 -to- 1 ratiometric threshold criteria method in 150/401 instances (37.4\%) and by the three-sigma statistical threshold criteria method in 259/401 instances $(64.6 \%)(\mathrm{P}<0.001)$.

Our current statistical analysis, which intentionally utilized a very large number of individual gamma detection probe measurement sets $(n=401)$ of presumed abnormal ${ }^{18}$ F-FDG-avid tissue sites that were evaluated completely independent of the count rate determinations by any specific type of gamma detection probe system, clearly demonstrates that the three-sigma statistical threshold criteria method was significantly better than the 1.5-to-1 ratiometric threshold criteria method for 


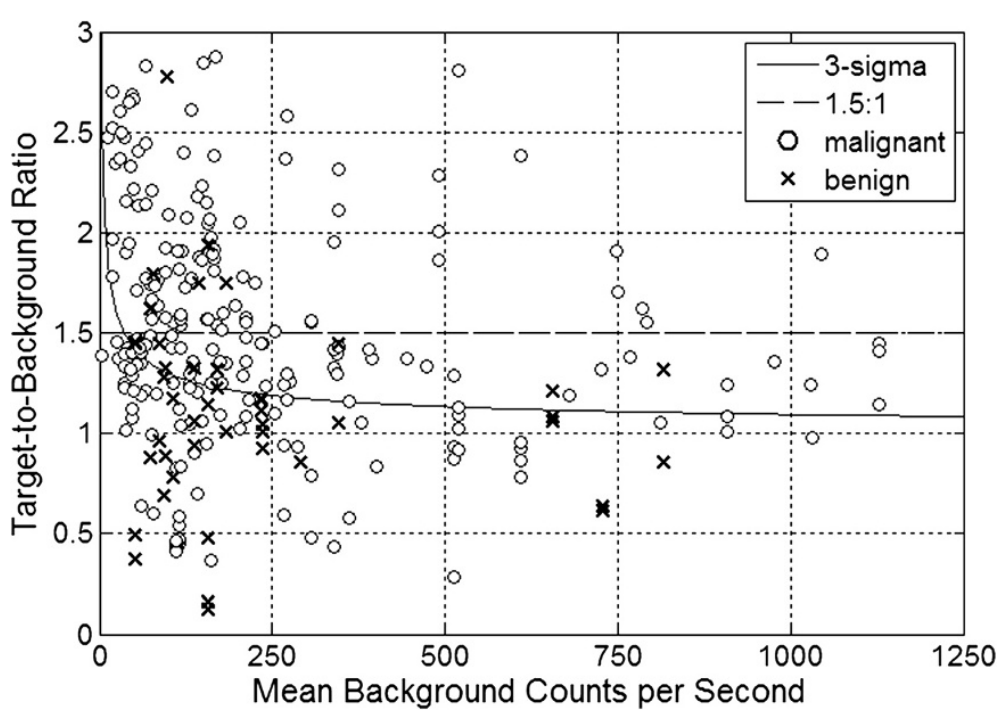

Figure 2 Plot of the target-to-background ratio and mean background count rate for those 291 of the 401 gamma detection probe measurement data sets that were limited to a mean background count rate range of less than 1250 counts per second. Malignant ${ }^{18} \mathrm{~F}-\mathrm{FDG}-$ avid tissue sites are shown as $(\boldsymbol{o})$ and benign ${ }^{18} \mathrm{~F}-\mathrm{FDG}$-avid tissue sites are shown as $(\boldsymbol{x})$. The thresholds for probe positivity, expressed as target-tobackground ratios, are graphed as a function of the mean background count rate. The ratiometric threshold criteria of 1.5-to-1 is shown as a dashed line and the three-sigma threshold criteria is shown as a solid line curve.

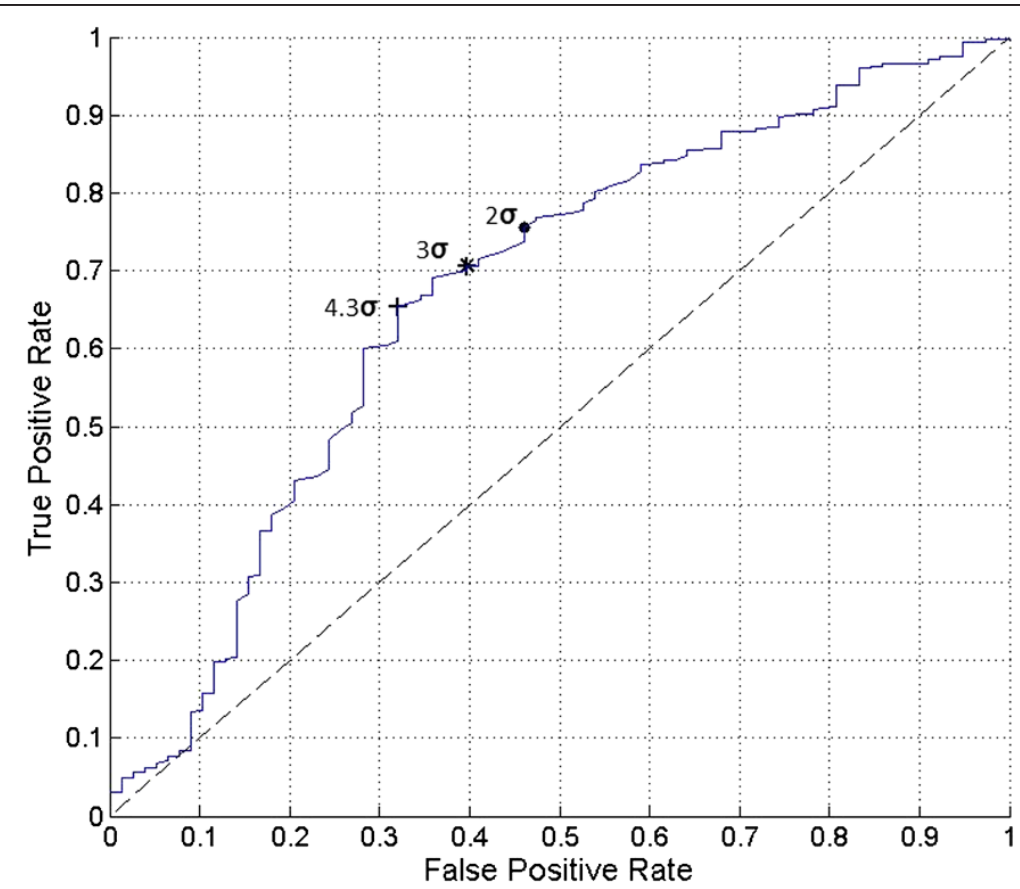

Figure 3 The receiver operating characteristic $(\mathrm{ROC})$ curve for various statistical threshold criteria values for probe positivity from the entire group of $\mathbf{4 0 1}$ gamma detection probe measurement sets is illustrated. The data curve is labeled with the specific numbers of standard deviations indicated. The dashed line indicates a hypothesis test of no diagnostic discrimination. The ROC curve ranges from -46 to 74 standard deviations above the mean background count rate. The operating point on the data curve for three standard deviations above the mean background count rate is indicated as three-sigma threshold criteria $(*)$, and corresponds to a true positive rate of $71 \%$ and false positive rate of $40 \%$. Two-sigma operating point is indicated by $(\bullet)$. The optimal operating point is indicated by (+). The area under the ROC curve is $0.6728(67.3 \%)$. 


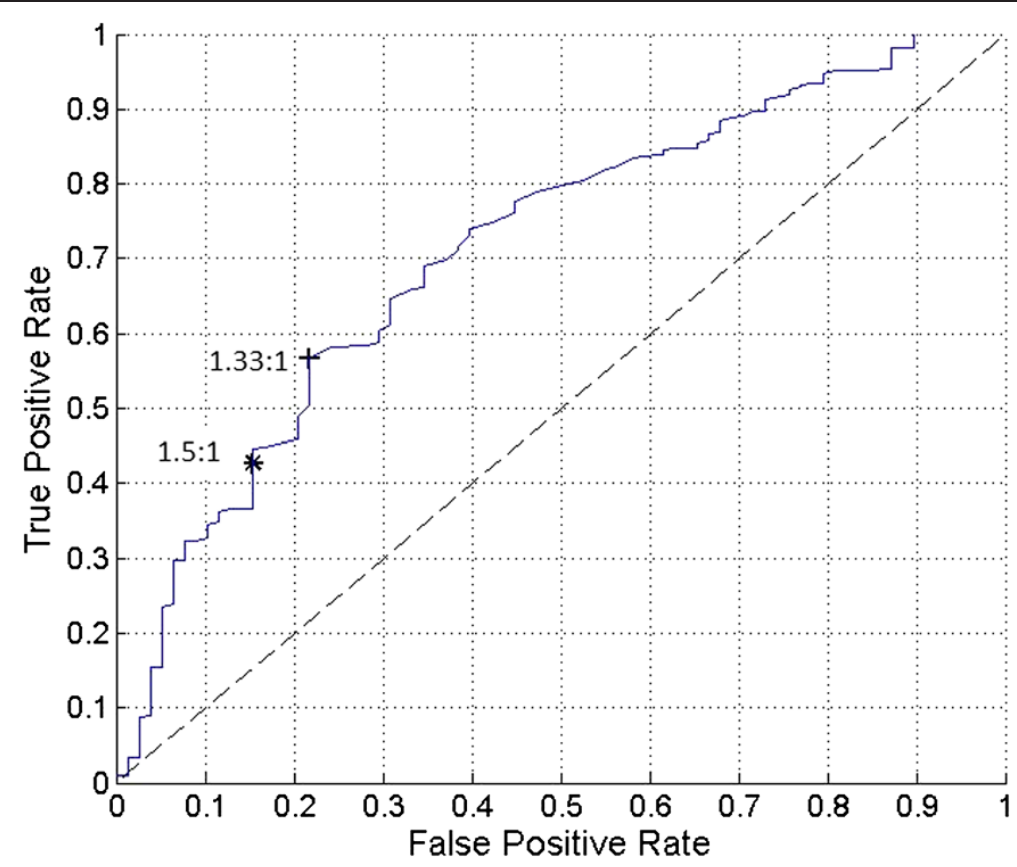

Figure 4 The receiver operating characteristic $(\mathrm{ROC})$ curve for various ratiometric threshold criteria values for probe positivity from the entire group of $\mathbf{4 0 1}$ gamma detection probe measurement sets is illustrated. The ROC curve ranges from target-to-background ratios of -1-to-1 to 9-to-1 above the mean background count rate. The 1.5 -to-1 ratiometric operating point is indicated by $\left(^{*}\right.$ ). The 1.33 -to-1 (optimal) ratiometric operating point is indicated by $(+)$. The area under the ROC curve is $0.7150(71.5 \%)$.

determination of gamma detection probe positivity for intraoperative in situ identification of presumed abnormal ${ }^{18} \mathrm{~F}$-FDG-avid tissue sites during ${ }^{18} \mathrm{~F}$-FDG-directed surgery.

The authors fully acknowledge and accept the wellestablished fact that the finding of ${ }^{18} \mathrm{~F}$-FDG-avidity within any given area of tissue is not an absolute indication that one will find the presence of malignancy within that given area of tissue. To further explore this subject matter, subset analysis was subsequently performed, as specifically based upon the postoperative histopathologic determination of the finding of malignant tissue versus benign tissue from all tissue specimens comprising the entire group of 401 gamma detection probe measurements sets initially analyzed. Table 1 shows the arbitrary breakdown of in situ probe positivity and in situ probe negativity versus the postoperative histopathologic determination of the finding of malignant tissue versus benign tissue. Of the 401 gamma detection probe measurements sets taken in situ from presumed abnormal ${ }^{18} \mathrm{~F}$-FDG-avid tissue

Table 1 Definitions for in situ probe positivity and in situ probe negativity versus the postoperative histopathologic determination of the finding of malignant tissue versus benign tissue

\begin{tabular}{lll}
\hline $\begin{array}{l}\text { Postoperative histopathologic } \\
\text { determination }\end{array}$ & $\begin{array}{l}\text { in situ probe } \\
\text { positive }\end{array}$ & $\begin{array}{l}\text { in situ probe } \\
\text { negative }\end{array}$ \\
\hline Malignant & True Positive (TP) & False Negative (FN) \\
Benign & False Positive (FP) & True Negative (TN) \\
\hline
\end{tabular}

sites, postoperative histopathologic evaluation identified 323 malignant tissue specimens and 78 benign tissue specimens. Figure 2 shows the plot of the target-tobackground ratio and mean background count rate for each malignant tissue site (o) and each benign tissue site (x) for those 291 of the 401 gamma detection probe measurement data points within the area of relevancy (i.e. around the ratiometric threshold criteria range of 1.5-to-1 for the target-to-background ratio and within the lower background count rate range of less than 1250 counts per second). Table 2 shows the frequency of true positives (TP), false positives (FP), true negatives (TN), and false negative (FN) versus threshold criteria method. Table 2 illustrates the increase in the number of malignant tissue sites identified as in situ probe positive using the three-sigma statistical threshold criteria method as compared to the 1.5-to-1 ratiometric threshold criteria method. As evident in Figure 2, there were numerous in situ probe positive measurement data points above the solid line curve for the three-sigma statistical threshold

Table 2 Frequency of true positives (TP), false positives (FP), true negatives (TN), and false negative (FN) versus threshold criteria method

\begin{tabular}{cccccccc}
\hline $\begin{array}{c}\text { 1.5-to-1 ratiometric threshold } \\
\text { criteria } \text { method }\end{array}$ & \multicolumn{4}{c}{$\begin{array}{c}\text { Three-sigma statistical threshold } \\
\text { criteria method }\end{array}$} \\
\hline TP & FP & TN & FN & TP & FP & TN & FN \\
138 & 12 & 66 & 185 & 228 & 31 & 47 & 95 \\
\hline
\end{tabular}


criteria curve and below the 1.5-to-1 ratiometric threshold criteria dashed line.

The solid line in Figure 2, representing the threesigma statistical threshold criteria curve, illustrates the advantage of the three-sigma statistical threshold criteria over that of the 1.5-to-1 ratiometric threshold criteria. Unlike the ratiometric threshold, in theory, the threesigma statistical threshold approaches a 1-to-1 target-tobackground ratio as the mean background count rate increases. For this reason, a gamma detection probe of higher counting efficiency is capable of detecting lower target-to-background ratios when the three-sigma statistical threshold criteria is applied. At increased mean background count rates, the three-sigma statistical threshold criteria can theoretically detect target-to-background ratios as low as 1.1-to-1 with a statistical confidence level of greater than $85.6 \%$, as previously shown in Figure 1 (i.e., probability of true positives) and in the derivation of three-sigma statistical threshold criteria method of probe positivity in the Methods section.

By contrast, in theory, the ratiometric threshold criteria method for determining probe positivity will result in an inconsistent statistical confidence level for target count rate measurements. The statistical confidence level of target count rate measurements is reduced at lower target count rates. As a result, the statistical confidence level for probe positivity when applying the ratiometric threshold criteria is an unknown variable at the time of in situ target count rate measurement. This is not the case when using statistical threshold criteria methods for probe positivity, since the statistical confidence level remains constant regardless of the count rate of the individual target count rate measurements.

The sensitivity (i.e., true positive rate) and the specificity (i.e., true negative rate) for the two threshold criteria methods (i.e., 1.5-to-1 ratiometric threshold criteria and three-sigma statistical threshold criteria) are shown in Table 3, and were calculated for the entire group of 401 gamma detection probe measurement sets using the postoperative histopathologic determination of malignant tissue versus benign tissue from Table 1 and the frequency of true positives (TP), false positives (FP), true negatives (TN), and false negative (FN) versus threshold criteria method from Table 2. The use of the three-sigma statistical threshold criteria method resulted in a $28 \%$ increase in sensitivity and

Table 3 Sensitivity and specificity calculations for each threshold criteria method

\begin{tabular}{ccc}
\hline $\begin{array}{c}\text { Statistical } \\
\text { metric }\end{array}$ & $\begin{array}{c}\text { 1.5-to-1 ratiometric } \\
\text { threshold criteria method }\end{array}$ & $\begin{array}{c}\text { Three-sigma statistical } \\
\text { threshold criteria method }\end{array}$ \\
\hline $\begin{array}{c}\text { Sensitivity } \\
\mathrm{TP} /(\mathrm{TP}+\mathrm{FN})\end{array}$ & 0.43 & 0.71 \\
Specificity & & \\
$\mathrm{TN} /(\mathrm{TN}+\mathrm{FP})$ & 0.85 & 0.60 \\
\hline
\end{tabular}

a $25 \%$ decrease in specificity. It is our contention that increased sensitivity of a detection probe system generally represents a much more influential variable for affecting long-term patient outcome than does the loss of specificity of a detection probe system. The advantage of the higher sensitivity is the allowance of fewer instances in which areas of malignant tissue will go undetected and inadvertently unresected within any given patient. The negative impact of a lower specificity (i.e., more false positive findings) is the increased frequency of possible unnecessary surgical excision of areas of nonmalignant tissue in any given patient. From a clinical perspective, inadvertently leaving unresected malignant tissues within any given patient represents a far greater risk to the patient than does surgically excising tissues from the patient that are later found to be nonmalignant. This premise is well-supported by long-term survival data from antigen-directed cancer surgery for patients with primary colorectal cancer $[63,64]$. For this reason, the three-sigma statistical threshold criteria method represents a marked improvement over the 1.5-to-1 ratiometric threshold criteria method for the successful intraoperative detection of malignant tissue.

In generally, the receiver operating characteristic (ROC) is a concept used in signal detection theory to illustrate the performance of a binary classification system as the discrimination threshold is varied [65-67]. In the current analysis, the ROC provides an alternative method for comparing true positive rate and false positive rate (1-specificity). Each point on any given ROC curve is the true positive rate (sensitivity) plotted against the false positive rate (1-specificity) resulting from the threshold for probe positivity calculated for a single value. At each new threshold for probe positivity, the sensitivity and specificity were recalculated. It is essential to understand that the ROC curve, as well as sensitivity and specificity, are measuring the predictive values of not only the threshold for probe positivity, but also the entire diagnostic system, including the specifications of the gamma detection probe system utilized, as well as the subjective selection by the surgeon of the background site and the target tissue sites for recording each measurement data set. Moreover, differing data sets result in some change to the ROC curve. However, within a single ROC curve, examining the effect of changing the threshold on sensitivity and specificity is valid, provided that the changes in the statistical confidence level for the analysis is also taken into consideration.

The ROC curve for various statistical threshold criteria values for probe positivity from the entire group of 401 gamma detection probe measurement sets is illustrated in Figure 3. Figure 3 illustrates the ROC of the current data set as the number of standard deviations was varied from a value of -46 to 74 in nonlinear increments to generate a complete curve. The ROC curve was generated by varying the number of standard deviations that must be exceeded 
above the mean background count rate for a probe positive threshold. The operating point on the data curve for three standard deviations above the mean background count rate (i.e., three-sigma statistical threshold) indicated by the " symbol in Figure 3, corresponds to a true positive rate of $71 \%$ and false positive rate of $40 \%$. Ideally, the true positive rate of the diagnostic test would be maximized (close to $100 \%$ ) and the false positive rate would be minimized (approaching 0\%) [65]. The current data set suggested that a statistical threshold using fewer than three standard deviations above the mean background count rate can increase the true positive rate without exceeding a $50 \%$ false positive rate. However, this modification would also result in a reduction in the statistical confidence level of the measurement. A statistical threshold for probe positivity of the mean background count rate plus two standard deviations, for example, would reduce the statistical confidence level of a true positive and true negative measurement from a $85.6 \%$ probability for three-sigma statistical threshold to a $76.0 \%$ probability for the for two-sigma statistical threshold.

It is also a common practice to identify the point on the ROC curve that represents the best combination of specificity and sensitivity [67]. In Figure 3, this point occurs at the location on the data curve that is closest to a true positive rate of a value of one and a false positive rate of a value of zero, which is located in the upper left hand corner of the plot. For the current data set shown in Figure 3, the best combination of specificity and sensitivity, as indicated by the + symbol, occurred at a true positive rate of $65 \%$ and false positive rate of $32 \%$, and corresponds to a probe positive threshold level of the mean background count rate plus 4.3 standard deviations. Although utilizing this approach for identifying an optimal operating point on the ROC curve is commonplace, the resultant sensitivity of $65 \%$ for any such diagnostic instrument which would be used for detecting ${ }^{18}$ F-FDG-avid tissue sites would be considered suboptimal and would make any such diagnostic instrument clinically ineffective. Therefore, it is our opinion that the three-sigma statistical threshold criteria (i.e., probe positive threshold level of the mean background count rate plus 3 standard deviations) for determination of gamma detection probe positivity represents a good balance of sensitivity, selectivity, and statistical confidence levels for positive and negative probabilities. This contention for our support of the threesigma statistical threshold criteria for determination of gamma detection probe positivity is further borne out in the fact that the three-sigma statistical threshold criteria is routinely used in other medical applications and commercial products that detect signals in the presence of background noise, such as radar detectors [68-70].

The ROC curve for various ratiometric threshold criteria values for probe positivity from the entire group of 401 gamma detection probe measurement sets is illustrated in
Figure 4. A similar analysis can be applied to the ratiometric threshold for probe positivity. Figure 4 illustrates the ROC of the current data set as the ratio for target-tobackground count rate for probe positivity is varied from -1-to-1 to 9-to-1. The optimal operating point the ratiometric threshold ROC curve occurred at a target-tobackground ratio of 1.33-to-1. It should be noted that this value validates our earlier contention that a target-tobackground ratio that is lower than 1.5-to-1 is required to adequately detect ${ }^{18} \mathrm{FDG}$-avid sites which are subsequently determined to be malignant.

In Table 4, we have summarized the frequency of true positives (TP), false positives (FP), true negatives (TN), and false negative (FN) as a function of four various probe positive threshold criteria, including (1) three-sigma statistical threshold (i.e., mean background count rate plus 3 standard deviations; (2) 4.3-sigma statistical threshold (i.e., mean background count rate plus 4.3 standard deviations; (3) target-to-background count rate ratio of 1.5-to-1 ratiometric threshold; and (4) target-to-background count rate ratio of 1.33-to-1 ratiometric threshold. The 1.33-to-1 ratiometric threshold represented a marked improvement over the 1.5-to-1 ratiometric threshold as the detection of true positives was increased from 138 to 183, and the frequency of false negative measurements was reduced from 185 to 140. Given the impact to patient outcome, improvements in these two parameters were more significant than the corresponding reduction in the frequency of true negatives and increase in the frequency of false positive measurements. Neither of the ratiometric threshold criteria values for probe positivity was as accurate in the correct detection of true positive and false negative sites as was either of the statistical threshold criteria values. Moreover, the threesigma statistical threshold criteria method provides the best outcome for detection of both true positive and false negative sites.

\section{Conclusion}

Our current data analysis demonstrates that the threesigma statistical threshold criteria method is significantly better than the 1.5-to-1 ratiometric threshold criteria method for determination of gamma detection probe positivity for intraoperative in situ detection of presumed abnormal ${ }^{18} \mathrm{~F}$-FDG-avid tissue sites during radioguided

Table 4 Frequency of true positives (TP), false positives (FP), true negatives (TN), and false negative (FN) as a function of four various probe positive threshold criteria

\begin{tabular}{lllll}
\hline Threshold criteria method & TP & FP & TN & FN \\
\hline 3-Sigma Statistical & 228 & 31 & 47 & 95 \\
4.3-Sigma Statistical & 211 & 25 & 53 & 112 \\
1.5-to-1 Ratiometric & 138 & 12 & 66 & 185 \\
1.33-to-1 Ratiometric & 183 & 17 & 61 & 140 \\
\hline
\end{tabular}


oncologic surgery. Moreover, the three-sigma statistical threshold criteria method can detect true positive results at target-to-background ratios that are much lower than the 1.5-to-1 target-to-background ratio of the 1.5-to-1 ratiometric threshold criteria method. If a surgeon utilizes a gamma detection probe system with high count rate sensitivity, it is theoretically feasible that target-to-background ratios as low as 1.1-to-1 can be identified as in situ probe positive ${ }^{18}$ F-FDG-avid tissue sites when applying the threesigma statistical threshold criteria method. This finding may be extremely important for reshaping the ongoing and future research and development of gamma detection probe systems that are necessary for optimizing the in situ detection of radioisotopes of higher-energy gamma photon emissions used during radioguided oncologic surgery.

\section{Competing interests}

The authors declare that they have no conflict of interests specifically related to the contents of this paper.

\section{Authors' contributions}

GJC and SPP contributed equally to this work. GJC was involved in the study design, data analysis and interpretation, writing of all drafts of the manuscript, and has approved final version of the submitted manuscript. SPP was the principle investigator for The Ohio State University Cancer IRB-approved, prospective, pilot study protocol involving patients undergoing ${ }^{18}$ F-FDG-directed surgery for known or suspected malignancy, and was responsible for the study design, data collection, data analysis and interpretation, writing of all drafts of the manuscript, and has approved final version of the submitted manuscript. NCH was involved in study design, critiquing drafts of the manuscript, and has approved final version of the submitted manuscript. DAM was involved in study design, data collection, data analysis, critiquing drafts of the manuscript, and has approved final version of the submitted manuscript. $\mathrm{RL}$ was involved in study design, critiquing drafts of the manuscript, and has approved final version of the submitted manuscript. EWM was involved in study design, critiquing drafts of the manuscript, and has approved final version of the submitted manuscript. All authors read and approved the final manuscript.

\section{Acknowledgements}

The authors would like to thank the following surgeons at The Ohio State University Wexner Medical Center for their utilization of ${ }^{18} \mathrm{~F}-\mathrm{FDG}$-directed surgery patients to this paper: Drs. Mark Bloomston, David Cohn, Amit Agrawal, David O'Malley, Enver Ozer, Carl Schmidt, Michael Walker, Susan Moffatt-Bruce, William Kraybill, and John Phay. The authors would like to thank Dr. Donn Young from the Center for Biostatistics of the Comprehensive Cancer Center at The Ohio State University Wexner Medical Center, Dr. Charles Hitchcock from the Department of Pathology at The Ohio State University Wexner Medical Center, and Dr. Marvin White of the Department of Electrical and Computer Engineering at The Ohio State University. The authors would like to thank Deborah Hurley, Marlene Wagonrod, and the entire staff of the Division of Nuclear Medicine from the Department of Radiology at The Ohio State University Wexner Medical Center, Nichole Storey from the Department of Radiology at The Ohio State University Wexner Medical Center, and the entire operating room staff from the Arthur G. James Cancer Hospital and Richard J. Solove Research Institute at The Ohio State University Wexner Medical Center.

\section{Author details}

'Department of Electrical and Computer Engineering, The Ohio State University, Columbus, $\mathrm{OH} 43210$, USA. ² Division of Surgical Oncology, Department of Surgery, Arthur G. James Cancer Hospital and Richard J. Solove Research Institute and Comprehensive Cancer Center, The Ohio State University Wexner Medical Center, Columbus, OH 43210, USA. ${ }^{3}$ Division of Molecular Imaging and Nuclear Medicine, Department of Radiology, The Ohio State University Wexner Medical Center, Columbus, OH 43210, USA.

Received: 7 July 2014 Accepted: 10 September 2014

Published: 13 September 2014

\section{References}

1. Povoski SP, Neff RL, Mojzisik CM, O'Malley DM, Hinkle GH, Hall NC, Murrey DA Jr, Knopp MV, Martin EW Jr: A comprehensive overview of radioguided surgery using gamma detection probe technology. World J Surg Oncol 2009, 7:11.

2. Povoski SP, Chapman GJ, Murrey DA Jr, Lee R, Martin EW Jr, Hall NC: Intraoperative detection of 18F-FDG-avid tissue sites using the increased probe counting efficiency of the K-alpha probe design and variance-based statistical analysis with the three-sigma criteria. BMC Cancer 2013, 13:98.

3. Desai D, Arnold M, Saha S, Hinkle G, Soble D, Frye J, DePalatis L, Mantil J, Satter M, Martin E: Intraoperative gamma detection of FDG distribution in colorectal cancer. Clin Positron Imaging 1999, 2:325.

4. Desai DC, Arnold M, Saha S, Hinkle G, Soble D, Fry J, DePalatis LR, Mantil J, Satter M, Martin EW: Correlative whole-body FDG-PET and intraoperative gamma detection of FDG distribution in colorectal cancer. Clin Positron Imaging 2000, 3:189-196.

5. Zervos EE, Desai DC, DePalatis LR, Soble D, Martin EW: 18F-labeled fluorodeoxyglucose positron emission tomography-guided surgery for recurrent colorectal cancer: a feasibility study. J Surg Res 2001, 97:9-13.

6. Essner R, Hsueh EC, Haigh PI, Glass EC, Huynh Y, Daghighian F: Application of an [(18)F]fluorodeoxyglucose-sensitive probe for the intraoperative detection of malignancy. J Surg Res 2001, 96:120-126.

7. Essner R, Daghighian F, Giuliano AE: Advances in FDG PET probes in surgical oncology. Cancer J 2002, 8:100-108.

8. Higashi T, Saga T, Ishimori T, Mamede M, Ishizu K, Fujita T, Mukai T, Sato S, Kato H, Yamaoka Y, Matsumoto K, Senda M, Konishi J: What is the most appropriate scan timing for intraoperative detection of malignancy using 18F-FDG-sensitive gamma probe? Preliminary phantom and preoperative patient study. Ann Nucl Med 2004, 18:105-114.

9. Yap JT, Carney JP, Hall NC, Townsend DW: Image-guided cancer therapy using PET/CT. Cancer J 2004, 10:221-233.

10. Barranger E, Kerrou K, Petegnief Y, David-Montefiore E, Cortez A, Daraï E: Laparoscopic resection of occult metastasis using the combination of FDG-positron emission tomography/computed tomography image fusion with intraoperative probe guidance in a woman with recurrent ovarian cancer. Gynecol Oncol 2005, 96:241-244

11. Carrera D, Fernandez A, Estrada J, Martin-Comin J, Gamez C: Detection of occult malignant melanoma by $18 \mathrm{~F}-\mathrm{FDG}$ PET-CT and gamma probe. Rev Esp Med Nucl 2005, 24:410-413. Spanish.

12. Franc BL, Mari C, Johnson D, Leong SP: The role of a positron- and high-energy gamma photon probe in intraoperative localization of recurrent melanoma. Clin Nucl Med 2005, 30:787-791.

13. Kraeber-Bodéré F, Cariou B, Curtet $C$, Bridji B, Rousseau C, Dravet F, Charbonnel B, Carnaille B, Le Néel JC, Mirallié E: Feasibility and benefit of fluorine 18-fluoro-2-deoxyglucose-guided surgery in the management of radioiodine-negative differentiated thyroid carcinoma metastases. Surgery 2005, 138:1176-1182.

14. Gulec SA, Daghighian F, Essner R: PET-probe. Evaluation of technical performance and clinical utility of a handheld high-energy gamma probe in oncologic surgery. Ann Surg Oncol 2006. Epub ahead of print.

15. Meller B, Sommer K, Gerl J, von Hof K, Surowiec A, Richter E, Wollenberg B, Baehre $M$ : High energy probe for detecting lymph node metastases with 18F-FDG in patients with head and neck cancer. Nuklearmedizin 2006, 45:153-159.

16. Nwogu C, Fischer G, Tan D, Glinianski M, Lamonica D, Demmy T: Radioguided detection of lymph node metastasis in non-small cell lung cancer. Ann Thorac Surg 2006, 82:1815-1820. discussion 1820.

17. Curtet C, Carlier T, Mirallié E, Bodet-Milin C, Rousseau C, Barbet J, Kraeber-Bodéré F: Prospective comparison of two gamma probes for intraoperative detection of 18F-FDG: in vitro assessment and clinical evaluation in differentiated thyroid cancer patients with iodine-negative recurrence. Eur J Nucl Med Mol Imaging 2007, 34:1556-1562.

18. Gulec SA, Hoenie E, Hostetter R, Schwartzentruber D: PET probe-guided surgery: applications and clinical protocol. World J Surg Oncol 2007, 5:65.

19. Gulec SA: PET probe-guided surgery. J Surg Oncol 2007, 96:353-357.

20. Hall NC, Povoski SP, Murrey DA, Knopp MV, Martin EW: Combined approach of perioperative ${ }^{18} \mathrm{~F}-\mathrm{FDG} \mathrm{PET} / \mathrm{CT}$ imaging and intraoperative ${ }^{18} \mathrm{~F}-\mathrm{FDG}$ handheld gamma probe detection for tumor localization and verification of complete tumor resection in breast cancer. World J Surg Oncol 2007, 5:143

21. Piert M, Burian M, Meisetschlager G, Stein HJ, Ziegler S, Nahrig J, Picchio M, Buck A, Siewert JR, Schwaiger M: Positron detection for the intraoperative 
localisation of cancer deposits. Eur J Nucl Med Mol Imaging 2007, 34:1534-1544

22. Sarikaya I, Povoski SP, Al-Saif OH, Kocak E, Bloomston M, Marsh S, Cao Z, Murrey DA, Zhang J, Hall NC, Knopp MV, Martin EW: Combined use of preoperative 18F FDG-PET imaging and intraoperative gamma probe detection for accurate assessment of tumor recurrence in patients with colorectal cancer. World J Surg Oncol 2007, 5:80.

23. Sun D, Bloomston M, Hinkle G, Al-Saif OH, Hall NC, Povoski SP, Arnold MW, Martin EW: Radioimmunoguided surgery (RIGS), PET/CT image-guided surgery, and fluorescence image-guided surgery: past, present, and future. J Surg Oncol 2007, 96:297-308.

24. Agrawal A, Hall NC, Ringel MD, Povoski SP, Martin EW Jr: Combined use of perioperative TSH-stimulated 18F-FDG PET/CT imaging and gamma probe radioguided surgery to localize and verify resection of iodine scan-negative recurrent thyroid carcinoma. Laryngoscope 2008, 118:2190-2194.

25. Cohn DE, Hall NC, Povoski SP, Seamon LG, Farrar WB, Martin EW Jr: Novel perioperative imaging with 18F-FDG PET/CT and intraoperative 18F-FDG detection using a handheld gamma probe in recurrent ovarian cancer. Gynecol Oncol 2008, 110:152-157.

26. Hall NC, Povoski SP, Murrey DA, Knopp MV, Martin EW: Bringing advanced medical imaging into the operative arena could revolutionize the surgical care of cancer patients. Expert Rev Med Devices 2008, 5:663-667.

27. Moffatt-Bruce SD, Povoski SP, Sharif S, Hall NC, Ross P Jr, Johnson MA, Martin EW Jr: A novel approach to positron emission tomography in lung cancer. Ann Thorac Surg 2008, 86:1355-1357.

28. Piert M, Carey J, Clinthorne N: Probe-guided localization of cancer deposits using [(18)F]fluorodeoxyglucose. Q J Nucl Med Mol Imaging 2008, 52:37-49.

29. Povoski SP, Hall NC, Martin EW, Walker MJ: Multimodality approach of perioperative ${ }^{18} \mathrm{~F}$-FDG PET/CT imaging, intraoperative ${ }^{18} \mathrm{~F}-\mathrm{FDG}$ handheld gamma probe detection, and intraoperative ultrasound for tumor localization and verification of resection of all sites of hypermetabolic activity in a case of occult recurrent metastatic melanoma. World J Surg Oncol 2008, 6:1.

30. Povoski SP, Sarikaya I, White WC, Marsh SG, Hall NC, Hinkle GH, Martin EW $\mathrm{Jr}$, Knopp MV: Comprehensive evaluation of occupational radiation exposure to intraoperative and perioperative personnel from 18F-FDG radioguided surgical procedures. Eur J Nucl Med Mol Imaging 2008, 35:2026-2034.

31. Murrey DA Jr, Bahnson EE, Hall NC, Povoski SP, Mojzisik CM, Young DC, Sharif S, Johnson MA, Abdel-Misih S, Martin EW Jr, Knopp MV: Perioperative (18)F-fluorodeoxyglucose-guided imaging using the becquerel as a quantitative measure for optimizing surgical resection in patients with advanced malignancy. Am J Surg 2009, 198:834-840.

32. Gollub MJ, Akhurst TJ, Williamson MJ, Shia J, Humm JL, Wong WD, Paty PB Guillem JG, Weiser MR, Temple LK, Dauer LT, Jhanwar SC, Kronman RE Montalvo CV, Miller AR, Larson SM, Margulis AR: Feasibility of ex vivo FDG PET of the colon. Radiology 2009, 252:232-239.

33. Molina MA, Goodwin WJ, Moffat FL, Serafini AN, Sfakianakis GN, Avisar E: Intra-operative use of PET probe for localization of FDG avid lesions. Cancer Imaging 2009, 9:59-62.

34. Hall NC, Povoski SP, Murrey DA, Martin EW Jr, Knopp MV: Ex vivo specimen FDG PET/CT imaging for oncology. Radiology 2010, 255:663-664.

35. Nalley C, Wiebeck K, Bartel TB, Bodenner D, Stack BC Jr: Intraoperative radiation exposure with the use of (18)F-FDG-guided thyroid cancer surgery. Otolaryngol Head Neck Surg 2010, 142:281-283.

36. de Jong JS, van Ginkel RJ, Slart RH, Lemstra CL, Paans AM, Mulder NH, Hoekstra HJ: FDG-PET probe-guided surgery for recurrent retroperitoneal testicular tumor recurrences. Eur J Surg Oncol 2010, 36:1092-1095.

37. Lee GO, Costouro NG, Groome T, Kashani-Sabet M, Leong SPL: The use of intraoperative PET probe to resect metastatic melanoma. BMJ Case Reports 2010. doi:10.1136/bcr.12.2009.2593.

38. García JR, Fraile M, Soler M, Bechini J, Ayuso JR, Lomeña F: PET/CT-guided salvage surgery protocol. Results with ROLL technique and PET probe. Rev Esp Med Nucl 2011, 30:217-222. Spanish.

39. Kim WW, Kim JS, Hur SM, Kim SH, Lee SK, Choi JH, Kim S, Choi JY, Lee JE, Kim JH, Nam SJ, Yang JH, Choe JH: Radioguided surgery using an intraoperative PET probe for tumor localization and verification of complete resection in differentiated thyroid cancer: a pilot study. Surgery 2011, 149:416-424.
40. Manca G, Biggi E, Lorenzoni A, Boni G, Roncella M, Ghilli M, Volterrani D, Mariani G: Simultaneous detection of breast tumor resection margins and radioguided sentinel node biopsy using an intraoperative electronically collimated probe with variable energy window: a case report. Clin Nucl Med 2011, 36:e196-e198.

41. Povoski SP, Hall NC, Murrey DA Jr, Chow AZ, Gaglani JR, Bahnson EE Mojzisik CM, Kuhrt MP, Hitchcock CL, Knopp MV, Martin EW Jr: Multimodal imaging and detection approach to 18F-FDG-directed surgery for patients with known or suspected malignancies: a comprehensive description of the specific methodology utilized in a single-institution cumulative retrospective experience. World J Surg Oncol 2011, 9:152.

42. Francis CL, Nalley C, Fan C, Bodenner D, Stack BC Jr: 18F-fluorodeoxyglucose and 1311 radioguided surgical management of thyroid cancer. Otolaryngol Head Neck Surg 2012, 146:26-32.

43. Bains S, Reimert M, Win AZ, Khan S, Aparici CM: A patient with psoriatic arthritis imaged with FDG-PET/CT demonstrated an unusual imaging pattern with muscle and fascia involvement: a case report. NuCl Med Mol Imaging 2012, 46:138-143.

44. Vos CG, Hartemink KJ, Muller S, Oosterhuis JW, Meijer S, van den Tol MP, Comans EF: Clinical applications of FDG-probe guided surgery. Acta Chir Belg 2012, 112:414-418.

45. Hall NC, Povoski SP, Zhang J, Knopp MV, Martin EW Jr: Use of intraoperative nuclear medicine imaging technology: strategy for improved patient management. Expert Rev Med Devices 2013, 10:149-152

46. Yasuda S, Makuuchi H, Fujii H, Nakasaki H, Mukai M, Sadahiro S, Tajima T, Ide $M$, Shohtsu A, Suzuki Y: Evaluation of a surgical gamma probe for detection of 18F-FDG. Tokai J Exp Clin Med 2000, 25:93-99.

47. Yamamoto S, Matsumoto K, Senda M: Optimum threshold setting for a positron-sensitive probe with background rejection capability. Ann Nucl Med 2004, 18:251-256

48. Yamamoto S, Matsumoto K, Sakamoto S, Tarutani K, Minato K, Senda M: An intra-operative positron probe with background rejection capability for FDG-guided surgery. Ann Nucl Med 2005, 19:23-28.

49. Yamamoto S, Higashi T, Matsumoto K, Senda M: Development of a positron-imaging detector with background rejection capability. Ann Nucl Med 2006, 20:655-662.

50. Thurston MO: Development of the gamma-detecting probe for radioimmunoguided surgery. In Radioimmunoguided Surgery (RIGS) in the Detection and Treatment of Colorectal Cancer. 1st edition. Edited by Martin EW. Austin: R.G. Landes Company; 1994:41-65.

51. Martin EW Jr, Thurston MO: The use of monoclonal antibodies (MAbs) and the development of an intraoperative hand-held probe for cancer detection. Cancer Invest 1996, 14:560-571.

52. Martin EW Jr, Thurston MO: Method for locating, differentiating, and removing neoplasms. Publication number: US 4782840 A. Filing date September 10, 1986. Publication date: November 8, 1988; http://www.google.com/patents/ US4782840.

53. Ramsey RC, Thurston MO: Gamma radiation detector with enhanced signal treatment. Publication number: US 4889991 A. Filing date: September 23, 1988. Publication date: December 26, 1989; http://www.google.com/patents/ US4889991.

54. Arnold MW, Young DC, Hitchcock CL, Schneebaum S, Martin EW Jr: Radioimmunoguided surgery in primary colorectal carcinoma: an intraoperative prognostic tool and adjuvant to traditional staging. Am J Surg 1995, 170:315-318.

55. Bertsch DJ, Burak WE Jr, Young DC, Arnold MW, Martin EW Jr: Radioimmunoguided Surgery system improves survival for patients with recurrent colorectal cancer. Surgery 1995, 118:634-638. discussion 638-639.

56. Bertsch DJ, Burak WE Jr, Young DC, Arnold MW, Martin EW Jr: Radioimmunoguided surgery for colorectal cancer. Ann Surg Oncol 1996, 3:310-316

57. Arnold MW, Hitchcock CL, Young DC, Burak WE Jr, Bertsch DJ, Martin EW Jr: Intra-abdominal patterns of disease dissemination in colorectal cancer identified using radioimmunoguided surgery. Dis Colon Rectum 1996, 39:509-513.

58. Barbera-Guillem E, Arnold MW, Nelson MB, Martin EW Jr: First results for resetting the antitumor immune response by immune corrective surgery in colon cancer. Am J Surg 1998, 176:339-343.

59. Martin EW, Chapman GJ, Subramaniam W, Povoski SP: Intraoperative detection of gamma emissions using K-alpha X-ray fluorescence. Expert Rev Med Devices 2010, 7:431-434. 
60. Currie LA: Limits for qualitative detection and quantitative determination. Application to radiochemistry. Anal Chem 1968, 40:586-593.

61. Knoll GF: Radiation Detection and Measurement. 4th edition. New York, New York: John Wiley \& Sons, Inc; 2010.

62. Miller I, Freund JE: Probability and Statistics for Engineers. 2nd edition. Englewood Cliffs, New Jersey: Prentice-Hall, Inc; 1977.

63. Povoski SP, Hatzaras IS, Mojzisik CM, Arnold MW, Hinkle GH, Hitchcock CL, Young DC, Martin EW Jr: Antigen-directed cancer surgery for primary colorectal cancer: 15-year survival analysis. Ann Surg Oncol 2012, 19:131-138.

64. Povoski SP, Hatzaras IS, Mojzisik CM, Martin EW Jr: Oncologic theranostics: recognition of this concept in antigen-directed cancer therapy for colorectal cancer with anti-TAG-72 monoclonal antibodies. Expert Rev Mol Diagn 2011, 11:667-670.

65. Green DM, Swets JA: Signal Detection Theory and Psychophysics. 1st edition. New York, New York: John Wiley \& Sons, Inc; 1966.

66. Zweig MH, Campbell G: Receiver-operating characteristic (ROC) plots: a fundamental evaluation tool in clinical medicine. Clin Chem 1993, 39:561-577.

67. Akobeng AK: Understanding diagnostic tests 3: receiver operating characteristic curves. Acta Paediatr 2007, 96:644-647.

68. Benneyan JC: Performance of number-between g-type statistical control charts for monitoring adverse events. Health Care Manag Sci 2001, 4:319-336.

69. Phillips GW, Nagel DJ, Coffey T: A Primer on the Detection of Nuclear and Radiological Weapons. 1st edition. Washington, DC: National Defense University Center for Technology and National Security Policy; 2005.

70. Jendzurski J, Paulter NG: Calibration of speed enforcement down-the-road radars. J Res Natl Inst Stand Technol 2009, 114:137-148.

doi:10.1186/1471-2407-14-667

Cite this article as: Chapman et al: Comparison of two threshold detection criteria methodologies for determination of probe positivity for intraoperative in situ identification of presumed abnormal ${ }^{18} \mathrm{~F}$-FDGavid tissue sites during radioguided oncologic surgery. BMC Cancer 2014 14:667.

\section{Submit your next manuscript to BioMed Central and take full advantage of:}

- Convenient online submission

- Thorough peer review

- No space constraints or color figure charges

- Immediate publication on acceptance

- Inclusion in PubMed, CAS, Scopus and Google Scholar

- Research which is freely available for redistribution 